\title{
Adverse effects of silver diamine fluoride treatment among preschool children
}

Short title: Adverse effect of silver diamine fluoride

\section{Duangthip D, Fung MHT, Wong MCM, LO ECM, Chu CH,}

Faculty of Dentistry, The University of Hong Kong, Hong Kong, China

Key words: Early childhood caries, fluoride, child, side effect, silver compounds

\begin{tabular}{|c|c|}
\hline Corresponding to: & CHU Chun Hung \\
\hline & 3/F Prince Philip Dental Hospital \\
\hline & Faculty of Dentistry \\
\hline & The University of Hong Kong \\
\hline & 34 Hospital Road \\
\hline & Hong Kong \\
\hline & Tel: +852 28590287 \\
\hline & Fax: +852 28582532 \\
\hline & E-mail: chchu@hku.hk \\
\hline
\end{tabular}

Number of words in abstract: 248

Number of words in text (abstract to acknowledgement): 3199

Number of tables: 4

Number of figures: 1

Number of cited references: 17 


\begin{abstract}
Objectives: This study aimed to compare the adverse effects and parental satisfaction following the different regimes of silver diamine fluoride $(S D F)$ treatment in preschool children.

Methods: A total of 888 preschool children who had active dentin caries received different SDF application regimes; Group 1-12\% SDF applied annually; Group 2-12\% SDF applied semi-annually; Group 3-38\% SDF applied annually; and Group 4-38\% SDF applied semiannually. Information on adverse effects including tooth or gum pain, gum swelling, gum bleaching and systemic toxicity were collected through a parental questionnaire after treatment every 6 months. Information of parental satisfaction on children's dental appearance was collected at baseline and 30-month examination.
\end{abstract}

Results: At the 30-month examination, 799 children (90\%) remained in the study. No acute systemic illness or major adverse effect was reported. No differences of all minor adverse effects among four groups were found ( $p>0.05)$. Overall, tooth and gum pain perceived by patients and reported by parents was 6.6\%, while gum swelling and gum bleaching was $2.8 \%$ and $4.7 \%$, respectively. Blackening of carious lesions was common in all groups with $36.7 \%$, 49.5\%, 65.6\% and 76.3\% in Groups 1-4, respectively ( $\chi 2$ test, $p<0.001$ ). The proportion of parental satisfaction with their child's dental appearance in Groups 1-4 were 67.6\%, 61.5\%, $70.8 \%$ and $62.3 \%$, respectively ( $\chi^{2}$ test, $p>0.05$ ).

Conclusion: SDF does not cause acute systemic illness. Tooth or gum pain, gum swelling and gum bleaching were uncommon and not significantly different among the study groups. Parental satisfaction with child's dental appearance was similar among four groups.

Clinical statement: The use of SDF following the study protocol for caries arrest is safe in preschool children. Collecting information on parental satisfaction and adverse effects are beneficial for dental professionals when deciding to adopt SDF treatment in preschool children.

This study was registered in the Registry of Clinical Trials run by the United States National Library of Medicine (NCT02385474). 


\section{Introduction}

The prevalence of early childhood caries (ECC) remains high in disadvantaged communities (Duangthip et al. 2017) Conventional restorative treatment may fail to solve the burden of ECC in many parts of the world. Most of the decayed teeth in preschool children remained unrestored (Chu et al. 2002). Recently, the use of silver diamine fluoride (SDF) has gained interest due to its caries-arrest effectiveness. Several clinical trials of SDF showed favourable results in arresting dental caries in preschool children (Fung et al. 2017, Duangthip et al. 2016). Systematic reviews concluded that the application of SDF can be a therapeutic option for caries management (Gao et al. 2016). Currently, SDF is marketed for reducing tooth sensitivity in the U.S.A. (Horst et al. 2016). Off-label use of SDF is now permissible for caries management in many countries. Although SDF treatment has been shown to be more efficacious when compared to other non-surgical approaches (Duangthip et al. 2015), the downsides of SDF in blackening carious lesions are of concern, probably affecting patients' acceptability. A survey on directors of paediatric residency programs in the U.S.A. found that poor parental acceptance was the most frequently reported barrier to use SDF (Nelson et al. 2016). Patient satisfaction is important as one of the proxy indicators representing the quality of health care. In young children, parent's satisfaction may play a major role affecting treatment adherence, resulting in the success of intervention. However, there is limited information in the current literature regarding these patient-based outcomes of the SDF studies.

Due to the high concentrations of fluoride and silver, the biocompatibility and adverse effect of SDF are of concern regarding its use in young children. Pulpal and tissue irritation are hypothesized side effects after SDF treatment. Previous clinical trials suggested that SDF did not cause severe pulpal damages (Chu et al. 2002). However, a few cases reported a mild and painful white lesion on the mucosa (Llodra et al. 2005). Gingival irritation may occur after SDF application and applying vaseline at the adjacent gingiva was suggested to prevent this (dos Santos et al. 2012). Different SDF application and different SDF concentrations have been proposed. Higher concentration of SDF was more effective than the lower concentration in arresting active caries (Fung et al. 2017). Questions remain if the higher concentration of SDF or the more frequency of SDF application will increase the possibility of adverse effects or lower the level of patient satisfaction. Results of SDF studies mainly focused on treatment effectiveness, whereas adverse effects were seldom systematically investigated. Despite the 
promising results of SDF, the American Dental Association Center for Evidence-Based Dentistry reported limited evidence regarding the use of SDF in children (Cheng 2017). Further clinical studies are required to fill the information gap regarding patient safety and preference.

The aim of this paper was to report on the adverse effects and parental satisfaction following the different regimes of SDF treatment in preschool children. The null hypothesis was that there is no difference in the adverse effects and parental satisfaction among four groups over 30 months.

\section{Materials and Methods}

Results of treatment effectiveness of this trial were published (Fung et al. 2017). This paper mainly presented the details of adverse effects and parental satisfaction of different SDF regimes. This trial was registered in the Registry of Clinical Trials run by the United States National Library of Medicine (ClinicalTrials.gov NCT02385474). Ethics approval was obtained from the Institutionalized Review Board of the University of Hong Kong. Written parental consent was obtained from all study children. The trial was implemented in 37 kindergartens in Hong Kong. The inclusion criteria were 3- to 4-year old children who had at least one cavitated dentine lesions. Children with severe systemic diseases were excluded.

The sample size calculation for this study was based on the caries arrest rate of SDF and the result showed that 565 caries lesions in 221 children in each group was required at baseline (Fung et al. 2017). After baseline examination, the study children with soft carious lesion(s) were randomly allocated to one of the four SDF treatment groups as follows:

Group1 - 12\%SDF applied once a year;

Group2 - 12\%SDF applied twice a year;

Group3 - 38\%SDF applied once a year;

Group4 - 38\%SDF applied twice a year.

Two concentrations of SDF solutions were used; 1) 12\% SDF solution (Cariestop Biodinâmica Química e Farmacêutica LTDA, Brazil), and 2) 38\% SDF solution (Saforide, Toyo Seiyaku Kasei Co. Ltd., Japan). An independent dentist who was blinded to the treatment groups applied SDF solution or placebo on the carious surfaces. For Groups 1 and 3, SDF was 
applied at baseline, 12- and 24-month while a normal saline solution was used at the 6-month, 18-month and 30-month. For Groups 2 and 4, SDF was applied every 6 months.

Steps of SDF application were as follows:

(1) position the study child supine on a table;

(2) remove food debris and plaque from the carious cavity;

(3) isolate the child's decayed teeth with dental gauze;

(4) apply the solution on each caries tooth surface with a micro applicator (Premium plus, Premium plus International Limited, Hong Kong) for approximately 1 minute;

(5) if necessary, soak away the excess solution with dental gauze;

(6) inform the class teacher that the study children should not eat or drink, or rinse their mouth for at least 30 minutes.

After treatment, an individual report on the child's oral health was submitted to parents. All clinical procedures were conducted in kindergartens. No parental involvement was required during the examination and treatment. Parents were invited to attend an oral health talk in the kindergarten once a year. No individual oral health counselling was provided.

Information about the study children' demographic information was collected by a close-ended structured questionnaire which was distributed to parents and then collected by class teachers after baseline examination. Follow-up examinations were performed every 6 months by a single examiner. Black staining of each lesion was clinically observed and recorded (yes/no). Information on adverse effects of SDF treatment including tooth or gum pain, gum swelling, gum bleaching and systemic toxicity were collected through a parental questionnaire at baseline and every 6 months throughout the 30 -month study period. Parent's satisfaction with their child's dental appearance and their child' dental health were collected through a self-rated parental questionnaire at baseline, 18- and 30-month follow up. If there was an urgent or acute systematic illness associated with SDF treatment, parents were informed to contact the principal investigator immediately by telephone.

The collected data were entered and analysed with the statistical software IBM SPSS 23.0 Statistics (SPSS Inc. Chicago, Illinois, USA). Analysis of variance (ANOVA) and chisquare test ( $\chi^{2}$ test) were performed to assess the comparability between four treatment groups according to the baseline conditions; continuous level variables and categorical variables, 
respectively. Chi-square test ( $\chi 2$ test) was used to assess for significant differences regarding the incidence of adverse effects and the parental satisfaction among four groups. Regarding the side effect of tooth staining, treatment group was replaced with SDF concentration $(12 \%$ or $38 \%$ ), frequency (annually or biannually). Logistic regression analysis was performed to analyse the effects of SDF concentration and frequency of application on the tooth staining at the 30-month examinations.

Regarding the parental satisfaction, logistic regression was used to evaluate the variables affecting the parental satisfaction at the 30-month follow up. The independent variables included the child's background characteristics (sex, place of birth, family structure, father and mother education level, family income) and clinical characteristics at baseline (no. of carious teeth treated with SDF, tooth type (anterior/posterior teeth). Backwards elimination method was adopted for modelling. McNemar test was performed to compare the differences in parental satisfaction of participant's dental appearance and dental health at baseline and the 30-month follow-up examination. The level of statistical significance was set at 0.05.

\section{Results}

A total of 4,251 preschool children were screened. Among them, 888 children (369 girls and 519 boys) participated in the study. At baseline, their mean age (SD) was 3.8(0.6) years. Their mean(SD) dmft and dmfs scores were 3.8(2.8) and 5.2(4.8) respectively. The mean(SD) number of decayed teeth that were included in the study were 3.7(2.7) with the range number between 1 and 15 . There was no statistically significant difference in the mean dmft and dmfs scores, and the number of included carious surfaces at baseline between groups (ANOVA, p>0.05). Regarding the demographic background, the two thirds of their fathers (66.2\%) and mothers $(69.8 \%)$ had just completed secondary education, while $17.3 \%$ of fathers and $12.9 \%$ of mothers had obtained a university degree. Around one third of the study children came from families with low monthly income. The four groups were comparable with respect to age (ANOVA, p>0.05), sex, place of birth, family structure, father and mother education levels and family income level ( $\chi 2$ test, $\mathrm{p}>0.05)$.

A CONSORT flow diagram during the 30-month follow-up period is shown in Figure 1. At the final examination, 799 children were evaluated, giving an overall dropout rate of $10 \%$. No significant differences in the dropout rates were found among four groups at all follow-up 
examinations ( $\chi^{2}$ test, $\mathrm{p}>0.05$ ). The main reason for leaving the study was moving to attend another kindergarten. All of the remaining study children returned the questionnaires at all follow-up examinations.

The distribution of tooth or gum pain, gum swelling and gum bleaching among groups is summarized in Table 1.

\section{Tooth or gum pain}

The number of study children complaining about tooth or gum pain/discomfort after treatment were $33(3.7 \%)$ at baseline, $44(5.0 \%)$ at 6-month, $55(6.2 \%)$ at 12-month, $62(7.0 \%)$ at 18-month, $60(6.8 \%)$ at 24-month, and $59(6.6 \%)$ at 30-month follow up. There was no significant difference in oral pain among groups at baseline and all the follow-up examinations $(\chi 2$ test, $p>0.05)$.

\section{Gum swelling}

The number of study children complaining about gum swelling after treatment were 19 (2.1\%), $13(1.5 \%), 26(2.9 \%), 22(2.5 \%), 22,(2.5 \%)$, and $25(2.8 \%)$ at baseline, 6-, 12-, 18-, 24-, and 30-month examination, respectively. No significant differences in gum swelling were found among four groups at all examinations ( $\chi^{2}$ test, $\left.\mathrm{p}>0.05\right)$.

\section{Gum bleaching}

The number of study children complaining about gum bleaching were 38 (4.3\%) at baseline, $49(5.5 \%)$ at 6-month, $45(5.1 \%)$ at 12-month, 27 (3.0\%) at 18-month, $51(5.7 \%)$ at 24-month, and $42(4.7 \%)$ at 30-month examination. All of the complaining children reported that gum bleaching was resolved without any treatment within 2 days. There were no statistically significant differences in gum bleaching among groups at baseline and all examinations ( $\chi 2$ test, $\mathrm{p}>0.05)$.

In addition, all the adverse effects above were not statistically related to the children's caries experience (dmft score) and the number of included teeth at baseline (ANOVA, $\mathrm{p}>0.05$ ).

\section{Systemic toxicity}

After receiving SDF at baseline and all follow-up examinations, none of the study children experienced any signs and symptoms associated with acute toxicity or systemic illness. 


\section{Black staining}

The proportion of treated carious lesions with black staining at 18- and 30-month follow-up is summarized in Table 2. At 30-month examination, out of the 3,268 carious lesions examined, $843(25.8 \%)$ carious lesions were active, whereas 2,425 (74.2\%) lesions were arrested. There were statistically significant differences in blackening carious lesions between four SDF groups at the 18- and 30-month examinations ( $\chi 2$ test, $\mathrm{p}<0.001)$. Lesions of the children receiving 38\% SDF had a higher chance of becoming black, compared to those treated with $12 \% \operatorname{SDF}(\mathrm{OR}=3.29$; 95\%CI: 2.84-3.81, $\mathrm{p}<0.001)$. Lesions treated semi-annually had a higher chance of becoming black in color, compared to those treated annually $(\mathrm{OR}=1.69$; 95\%CI: 1.46-1.95, $\mathrm{p}<0.001)$.

\section{Parental satisfaction with their child's dental appearance and dental health}

Parents' satisfaction with their children's dental appearance and dental health is shown in Table 3 and 4, respectively. No statistically significant differences in the satisfaction levels with child's dental appearance and dental health were found among four groups at all examinations ( $\chi^{2}$ test, $\mathrm{p}>0.05$ ). Comparing with the baseline findings, there were significant improvements in the parental satisfaction levels of both dental appearance and dental health at 18- and 30-month examinations (McNemar test, $\mathrm{p}<0.001$ ).

Regarding the logistic regression model of parental satisfaction with child' dental appearance at the 30-month follow-up, the two significant variables remaining in the final model were the level of monthly family income $(\mathrm{p}<0.001)$ and tooth type involved in the treatment (anterior or posterior teeth) $(\mathrm{p}<0.007)$. Compared with parents from the low income level (reference group), parents from the high and middle income level were not likely to be satisfied with their child's dental appearance (middle income level: OR 0.47; 95\%CI: 0.320.70 and high income level: OR: 0.50; 95\%CI: 0.34-0.75). Parents of children with decayed anterior teeth had a lower chance to be satisfied with their children's dental appearance, compared to those of children with decayed posterior teeth (OR: 0.54 ; 95\% CI: $0.35-0.84$ ).

Regarding the parental satisfaction with child's dental health, mother's education level was the only significant variable remained in the final model $(\mathrm{p}=0.001)$, while the other factors were not statistically significant. Compared with mothers who completed primary education level (reference group), mothers with secondary and tertiary education level were less likely to 
be satisfied with their children' dental health (secondary education level, OR: 0.33 ; 95\%CI 0.19-0.60 and tertiary education level, OR: 0.56, 95\% CI: 0.36-0.87).

\section{Discussion}

In previous studies, clinical outcomes of SDF treatment based on the clinicians' judgement were usually reported [Fung et al. 2017, dos Santos et al. 2014], while patientreported outcomes were not. Results of the present study add new knowledge regarding the safety of SDF treatment in preschool children. No incidence of serious adverse effects/systematic illness related to SDF application occurred during the whole study period. These results are in accordance with a previous study reporting no major side effects in children (Duangthip et al. 2016). Oral pain, transient gum swelling and gum bleaching were seldom reported in the present study. In summary, there is a minimal risk of developing minor side effects after SDF application over 30 months.

According to the treatment assignment, children in Group 1 and 3 received SDF treatment at baseline, 12- and 24-month follow up, while placebo was applied at the 6-, 18- and 30-month. Due to the blinding procedure, follow-up questionnaires were completed by all participants. Interestingly, similar proportion of the parents of children in Group 1 and 3 reported their child had adverse effects at the follow up which their child only received placebo. It is difficult to identify a complaint of pain or discomfort is an adverse effect of SDF treatment or if it is caused by underlying dental disease. Nevertheless, the present results could give an overall idea about the possibility of parent-reported minor adverse effects when implementing a SDF treatment program in kindergarten children.

Regarding the toxicity of fluoride, the average amount of fluoride was $0.33 \mathrm{mgF}$ in each application of 38\% SDF (Vasquez et al. 2012). The 'probably toxic dose' (PTD) of fluoride is suggested as $5 \mathrm{mg} \mathrm{F/kg} \mathrm{body} \mathrm{weight} \mathrm{(Whitford} \mathrm{1987).} \mathrm{The} \mathrm{weight} \mathrm{of} \mathrm{a} \mathrm{small} \mathrm{3-year} \mathrm{old} \mathrm{child}$ can be as low as $10 \mathrm{~kg}$. In the worst-case scenario, a child of $10 \mathrm{~kg}$ who has 20 decayed teeth will receive a maximum dose of $6.6 \mathrm{mgF}(0.33 \mathrm{mgF}$ x 20 teeth $)$ or the highest dose of fluoride would be $0.66 \mathrm{mgF} / \mathrm{kg}$. These would still be an 8 -fold safety margin of fluoride when applying SDF to all the primary teeth in a small child. 
Health risk associated with silver absorption (Ag+) is low (Lansdown 2010). For the safety of exposure to silver, the average $\mathrm{LD}_{50}$ of silver by oral and subcutaneous administration observed in rat studies was suggested to be $520 \mathrm{mg} / \mathrm{kg}$ and $380 \mathrm{mg} / \mathrm{kg}$, respectively (Horst et al. 2016). The amount of $38 \%$ SDF applied to the 3 teeth was reported to be $7.6 \mathrm{mg}$, which means that the amount of silver applied to the 3 teeth was approximately $1.50 \mathrm{mg}$ (Vasquez et al. 2012). In other words, the amount of silver applied on each decayed tooth was $0.5 \mathrm{mg}$. Thus, the maximum amount of silver applied on 20 decayed primary teeth would be approximately $10 \mathrm{mg}(0.5 \mathrm{mgAg} \times 20$ decayed teeth). If a preschool child of $10 \mathrm{~kg}$ is treated with $38 \% \mathrm{SDF}$, the highest dose of silver would be $10 \mathrm{mg}$ or $1 \mathrm{mg} / \mathrm{kg}$. If the $\mathrm{LD}_{50}$ by subcutaneous route is taken as reference, then, the safety margin of applying SDF on all decayed teeth of this child would be 380 -fold.

The appearance of black stains on SDF treated lesions was commonly found in this study, particularly, in the groups of higher SDF concentration and more frequent application. The clinical success of SDF application in arresting active caries lesion may be positively correlated with the presence of a protective layer, appearing as black staining over the lesion (Mei et al. 2014). Despite of the black stains on some of the SDF treated teeth, the proportion of parents who were satisfied with their child's dental appearance at the 30-month examination was higher than that at baseline. This may be because patient satisfaction is complex and multidimensional. Other aspects such as tooth alignment and dental caries status may also influence patient's satisfaction. Parent's social class (mother's education level and family income) significantly contributed to parental satisfaction in the present study. As parents with lower socio-economic status were more likely to be satisfied with the treatment outcome, this implies that the use of SDF would be more acceptable among parents in disadvantaged communities in which early childhood caries is more prevalent.

Although the current study had several strengths such as large sample size, low dropout rate and long follow up period, some limitations should be noted. First, information on the adverse effects was collected through a parent-reported questionnaire. There may be discrepancies between clinical findings and patients' report. Nevertheless, it would be very difficult to monitor the adverse effects closely by conducting frequent clinical examination of the study children. Second, if the adverse effect is mild, it may not be easily noticed by the young children and their parents. It should be noted that this study was conducted in generally 
healthy preschool children, these results may be not generalized to children with systematic diseases or very young children such as infants.

This evidence-based information regarding the safety and parental satisfaction would be valuable for dental health professionals when deciding to develop oral health program using SDF in kindergartens. Since the adverse effects are not significantly associated with the SDF application protocols, the adoption of which protocol should be based on its caries-arrest effectiveness. Whether the carious lesions are in the aesthetic zone of the mouth and children's family background are factors that need to be taken into account in the management of ECC with SDF treatment.

To conclude, caries arrest treatment by SDF is safe in preschool children. SDF does not cause acute or serious systemic illness. Report on oral pain, gum swelling and gum bleaching are rare and probably not related to the application of SDF. Staining on arrested carious lesions is common and more so with higher concentration and higher frequency of application of SDF. Despite this, parental satisfaction with their child's dental appearance is high and unrelated to the SDF application protocol. Further study on parental acceptability of SDF in countries with different cultures is required to confirm or refute these findings.

\section{Acknowledgements}

The authors declare no conflicts of interest. This clinical trial was supported by the Research Grants Council of Hong Kong (\#765213M). 
Table 1: Minor adverse effects experienced by the study children after treatments

\begin{tabular}{|c|c|c|c|c|c|}
\hline & $\begin{array}{c}\text { Group } 1 \\
(12 \%, \text { annual }) \\
(\mathrm{n}=222)\end{array}$ & $\begin{array}{c}\text { Group } 2 \\
(12 \%, \text { biannual }) \\
(\mathrm{n}=222)\end{array}$ & $\begin{array}{c}\text { Group } 3 \\
(38 \%, \text { annual }) \\
(\mathrm{n}=222)\end{array}$ & $\begin{array}{c}\text { Group4 } \\
(38 \%, \text { biannual }) \\
(n=222)\end{array}$ & p-value \\
\hline \multicolumn{6}{|c|}{ Tooth or gum pain } \\
\hline Baseline & $6(2.7 \%)$ & $4(1.8 \%)$ & $12(5.4 \%)$ & $11(5.0 \%)$ & 0.277 \\
\hline 6-month & $13(5.9 \%)^{*}$ & $12(5.4 \%)$ & $10(4.5 \%)^{*}$ & $9(4.1 \%)$ & 0.558 \\
\hline 12-month & $12(5.4 \%)$ & $17(7.7 \%)$ & $15(6.8 \%)$ & $11(5.0 \%)$ & 0.932 \\
\hline 18-month & $12(5.4 \%)^{*}$ & $10(4.5 \%)$ & $17(7.7 \%)^{*}$ & $23(10.4 \%)$ & 0.176 \\
\hline 24-month & $15(6.8 \%)$ & $13(5.9 \%)$ & $19(8.6 \%)$ & $13(5.9 \%)$ & 0.642 \\
\hline 30-month & $10(4.5 \%)^{*}$ & $11(5.0 \%)$ & $18(8.1 \%)^{*}$ & $20(9.0 \%)$ & 0.362 \\
\hline \multicolumn{6}{|c|}{ Gum swelling } \\
\hline Baseline & $0(0 \%)$ & $4(1.8 \%)$ & $9(4.1 \%)$ & $6(2.7 \%)$ & 0.155 \\
\hline 6-month & $4(1.8 \%)^{*}$ & $5(2.3 \%)$ & $2(0.9 \%)^{*}$ & $2(0.9 \%)$ & 0.328 \\
\hline 12-month & $6(2.7 \%)$ & $6(2.7 \%)$ & $7(3.2 \%)$ & $7(3.2 \%)$ & 0.81 \\
\hline 18-month & $4(1.8 \%)^{*}$ & $7(3.2 \%)$ & $3(1.4 \%)^{*}$ & $8(3.6 \%)$ & 0.36 \\
\hline 24-month & $6(2.7 \%)$ & $3(1.4 \%)$ & $6(2.7 \%)$ & $7(3.2 \%)$ & 0.896 \\
\hline 30-month & $7(3.2 \%)^{*}$ & $5(2.3 \%)$ & $7(3.2 \%)^{*}$ & $6(2.7 \%)$ & 0.519 \\
\hline \multicolumn{6}{|c|}{ Gum bleaching } \\
\hline Baseline & $7(3.2 \%)$ & $11(5.0 \%)$ & $11(5.0 \%)$ & $9(4.1 \%)$ & 0.923 \\
\hline 6-month & $11(5.0 \%)^{*}$ & $15(6.8 \%)$ & $9(4.1 \%)^{*}$ & $14(6.3 \%)$ & 0.691 \\
\hline 12-month & $12(5.7 \%)$ & $13(6.1 \%)$ & $10(4.7 \%)$ & $10(4.7 \%)$ & 0.72 \\
\hline 18-month & $5(2.4 \%)^{*}$ & $6(2.9 \%)$ & $10(4.8 \%)^{*}$ & $6(2.9 \%)$ & 0.748 \\
\hline 24-month & $13(6.5 \%)$ & $16(7.8 \%)$ & $9(4.5 \%)$ & $13(6.6 \%)$ & 0.901 \\
\hline 30-month & $12(6.1 \%)^{*}$ & $11(5.4 \%)$ & $7(3.5 \%)^{*}$ & $12(6.1 \%)$ & 0.826 \\
\hline
\end{tabular}

* For Groups 1 and 3, placebo (normal saline) was applied at the 6-, 18- and 30-month follow ups. 
Table 2: Color of carious surfaces at the 18-month and 30-month follow-up examinations

\begin{tabular}{|c|c|c|c|c|c|}
\hline & $\begin{array}{c}\text { Group } 1 \\
(12 \%, \text { annual })\end{array}$ & $\begin{array}{c}\text { Group } 2 \\
(12 \%, \text { biannual) }\end{array}$ & $\begin{array}{c}\text { Group3 } \\
(38 \% \text {, annual) }\end{array}$ & $\begin{array}{c}\text { Group } 4 \\
\text { (38\%, biannual) }\end{array}$ & p-value \\
\hline 18-month follow-up & & & & & $<0.001$ \\
\hline Active non-black & $393(44.7 \%)$ & $364(39.1 \%)$ & $302(31.8 \%)$ & $227(24.5 \%)$ & \\
\hline Arrested non-black & $183(20.8 \%)$ & $160(17.2 \%)$ & $67(7.0 \%)$ & $68(7.3 \%)$ & \\
\hline Arrested black & $304(34.5 \%)$ & $406(43.7 \%)$ & $582(61.2 \%)$ & $633(68.2 \%)$ & \\
\hline Total & $880(100 \%)$ & $930(100 \%)$ & $951(100 \%)$ & $928(100 \%)$ & \\
\hline 30-month follow-up & & & & & $<0.001$ \\
\hline Active non-black & $264(34 \%)$ & $253(30.4 \%)$ & $193(22.9 \%)$ & $134(16.4 \%)$ & \\
\hline Arrested non-black & $227(29.3 \%)$ & $167(20.1 \%)$ & $97(11.5 \%)$ & $60(7.3 \%)$ & \\
\hline Arrested black & $285(36.7 \%)$ & $411(49.5 \%)$ & $553(65.6 \%)$ & $625(76.3 \%)$ & \\
\hline Total & $776(100 \%)$ & $831(100 \%)$ & $843(100 \%)$ & $819(100 \%)$ & \\
\hline
\end{tabular}


Table 3: Parental satisfaction with children's dental appearance at baseline, 18-month and 30month follow-up

\begin{tabular}{|c|c|c|c|c|c|}
\hline & $\begin{array}{c}\text { Group } 1 \\
\text { (12\%, annual) }\end{array}$ & $\begin{array}{c}\text { Group } 2 \\
(12 \%, \text { biannual) }\end{array}$ & $\begin{array}{c}\text { Group3 } \\
\text { (38\%, annual) }\end{array}$ & $\begin{array}{c}\text { Group } 4 \\
(38 \%, \text { biannual) }\end{array}$ & p-value \\
\hline Baseline & & & & & 0.73 \\
\hline Very satisfied & $1(0.5 \%)$ & $2(0.9 \%)$ & $3(1.4 \%)$ & $1(0.5 \%)$ & \\
\hline Satisfied & $79(35.6 \%)$ & $84(37.8 \%)$ & $98(44.1 \%)$ & $97(43.7 \%)$ & \\
\hline Not satisfied & $98(44.1 \%)$ & $99(44.6 \%)$ & $91(41.0 \%)$ & $93(41.9 \%)$ & \\
\hline Very not satisfied & $8(3.6 \%)$ & $7(3.2 \%)$ & $5(2.3 \%)$ & $5(2.3 \%)$ & \\
\hline Not sure & $36(16.2 \%)$ & $30(13.5 \%)$ & $25(11.3 \%)$ & $26(11.7 \%)$ & \\
\hline 18-month follow-up & & & & & 0.365 \\
\hline Very satisfied & $15(7.2 \%)$ & $12(5.7 \%)$ & $6(2.9 \%)$ & $9(4.3 \%)$ & \\
\hline Satisfied & $128(61.8 \%)$ & $117(55.7 \%)$ & $131(63.3 \%)$ & $132(63.8 \%)$ & \\
\hline Not satisfied & $38(18.4 \%)$ & $51(24.3 \%)$ & $35(16.9 \%)$ & $41(19.8 \%)$ & \\
\hline Very not satisfied & $4(1.9 \%)$ & $4(1.9 \%)$ & $6(2.9 \%)$ & $7(3.4 \%)$ & \\
\hline Not sure & $22(10.6 \%)$ & $26(12.4 \%)$ & $29(14.0 \%)$ & $18(8.7 \%)$ & \\
\hline 30-month follow-up & & & & & 0.369 \\
\hline Very satisfied & $8(4.0 \%)$ & $7(3.4 \%)$ & $9(4.5 \%)$ & $8(4.1 \%)$ & \\
\hline Satisfied & $126(63.6 \%)$ & $118(58.1 \%)$ & $134(66.3 \%)$ & $114(58.2 \%)$ & \\
\hline Not satisfied & $34(17.2 \%)$ & $52(25.6 \%)$ & $32(15.8 \%)$ & $47(24.0 \%)$ & \\
\hline Very not satisfied & $9(4.5 \%)$ & $4(2.0 \%)$ & $3(1.59 \%)$ & $6(3.1 \%)$ & \\
\hline Not sure & $21(10.6 \%)$ & $22(10.8 \%)$ & $24(11.9 \%)$ & $21(10.7 \%)$ & \\
\hline
\end{tabular}


Table 4: Parental satisfaction with children's dental health at baseline, 18-month and 30-month follow-up

\begin{tabular}{|c|c|c|c|c|c|}
\hline & $\begin{array}{c}\text { Group } 1 \\
(12 \%, \text { annual) }\end{array}$ & $\begin{array}{c}\text { Group } 2 \\
(12 \%, \text { biannual })\end{array}$ & $\begin{array}{c}\text { Group3 } \\
\text { (38\%, annual) }\end{array}$ & $\begin{array}{c}\text { Group } 4 \\
\text { (38\%, biannual) }\end{array}$ & p-value \\
\hline Baseline & & & & & 0.171 \\
\hline Very satisfied & $0(0 \%)$ & $2(0.9 \%)$ & $3(1.4 \%)$ & $0(0 \%)$ & \\
\hline Satisfied & $57(25.7 \%)$ & $79(35.6 \%)$ & $64(28.8 \%)$ & $69(31.1 \%)$ & \\
\hline Not satisfied & $106(47.7 \%)$ & $107(48.2 \%)$ & $107(48.2 \%)$ & $102(45.9 \%)$ & \\
\hline Very not satisfied & $7(3.2 \%)$ & $5(2.3 \%)$ & $5(2.3 \%)$ & $6(2.7 \%)$ & \\
\hline Not sure & $52(23.4 \%)$ & $29(13.1 \%)$ & $43(19.4 \%)$ & $45(20.3 \%)$ & \\
\hline 18-month follow-up & & & & & 0.639 \\
\hline Very satisfied & $12(5.8 \%)$ & $9(4.3 \%)$ & $13(6.3 \%)$ & $12(5.8 \%)$ & \\
\hline Satisfied & $138(66.7 \%)$ & $141(67.1 \%)$ & $141(68.1 \%)$ & $144(69.6 \%)$ & \\
\hline Not satisfied & $27(13.0 \%)$ & $23(11.0 \%)$ & $17(8.2 \%)$ & $27(13.0 \%)$ & \\
\hline Very not satisfied & $2(1.0 \%)$ & $5(2.4 \%)$ & $2(1.0 \%)$ & $1(0.5 \%)$ & \\
\hline Not sure & $28(13.5 \%)$ & $32(15.2 \%)$ & $34(16.4 \%)$ & $23(11.1 \%)$ & \\
\hline 30-month follow-up & & & & & 0.877 \\
\hline Very satisfied & $10(5.1 \%)$ & $9(4.4 \%)$ & $6(3.0 \%)$ & $8(4.1 \%)$ & \\
\hline Satisfied & $107(54.0 \%)$ & $113(55.7 \%)$ & $125(61.9 \%)$ & $114(58.2 \%)$ & \\
\hline Not satisfied & $49(24.7 \%)$ & $48(23.6 \%)$ & $38(18.8 \%)$ & $39(19.9 \%)$ & \\
\hline Very not satisfied & $7(3.5 \%)$ & $4(2.0 \%)$ & $4(2.0 \%)$ & $7(3.6 \%)$ & \\
\hline Not sure & $25(12.6 \%)$ & $29(14.3 \%)$ & $29(14.4 \%)$ & $28(14.3 \%)$ & \\
\hline
\end{tabular}




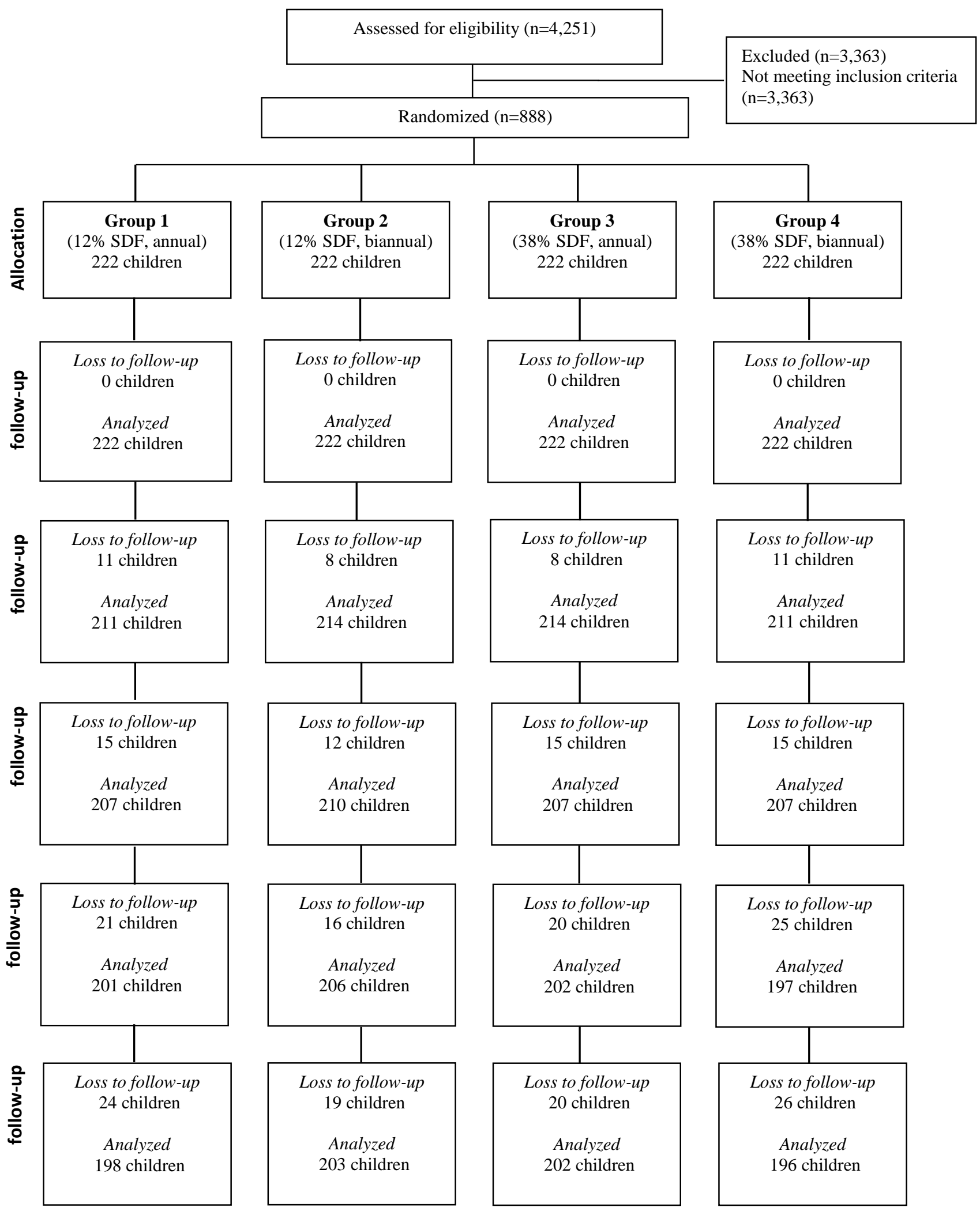

Figure 1 Flow chart of study children's progress over 30 months 


\section{References}

1. Chu CH, Ho PL, Lo EC. 2012. Oral health status and behaviours of preschool children in Hong Kong. BMC Public Health. 12:767.

2. Chu CH, Lo ECM, Lin HC. 2002. Effectiveness of silver diamine fluoride and sodium fluoride varnish in arresting dentin caries in Chinese pre-school children. J Dent Res. 81(11):767-770.

3. Cheng LL. 2017. Limited evidence suggesting silver diamine fluoride may arrest dental caries in children. J Am Dent Assoc. 148(2):120-122.

4. dos Santos VE Jr, de Vasconcelos FM, Ribeiro AG, Rosenblatt A. 2012. Paradigm shift in the effective treatment of caries in schoolchildren at risk. Int Dent J. 62(1): 47-51.

5. dos Santos VE Jr, de Vasconcelos FA, Targino AG, Flores MA, Galembeck A, Caldas AF Jr, Rosenblatt A. 2014. A new "silver-bullet" to treat caries in children--nano silver fluoride: a randomised clinical trial. J Dent. 42(8):945-951.

6. Duangthip D, Chu CH, Lo ECM. 2016. A randomized clinical trial on arresting dentine caries in preschool children by topical fluorides-18 month results. J Dent. 44:57-63.

7. Duangthip D, Gao SS, Lo ECM, Chu CH. 2017. Early childhood caries in 5-to 6- year old children in Southeast Asia. Int Dent J. 67(2):98-106.

8. Duangthip D, Jiang M, Chu CH, Lo ECM. 2015. Non-surgical treatment of dentin caries in preschool children - systematic review. BMC Oral Health. 15:44.

9. Fung MHT, Duangthip D, Wong MCM, Lo ECM, Chu CH. 2017. Randomized clinical trial of $12 \%$ and $38 \%$ silver diamine fluoride treatment. J Dent Res. Aug 1:22034517728496. doi: $10.1177 / 0022034517728496$.

10. Gao SS, Zhang S, Mei ML, Lo EC, Chu CH. 2016. Caries remineralisation and arresting effect in children by professionally applied fluoride treatment - a systematic review. BMC Oral Health.16:12.

11. Horst JA, Ellenikiotis H, Milgrom PL. 2016. UCSF Protocol for caries arrest using silver diamine fluoride: rationale, indications and consent. J Calif Dent Assoc. 44(1):16-28.

12. Lansdown AB. 2010. A pharmacological and toxicological profile of silver as an antimicrobial agent in medical devices. Adv Pharmacol Sci. 2010: 910686.

13. Llodra JC, Rodriguez A, Ferrer B, Menardia V, Ramos T, Morato M. 2005. Efficacy of silver diamine fluoride for caries reduction in primary teeth and first permanent molars of schoolchildren: 36-month clinical trial. J Dent Res. 84(8):721-724. 
14. Mei ML, Ito L, Cao Y, Lo EC, Li QL, Chu CH. 2014. An ex vivo study of arrested primary teeth caries with silver diamine fluoride therapy. J Dent. 42(4):395-402.

15. Nelson T, Scott JM, Crystal YO, Berg JH, Milgrom P. 2016. Silver diamine fluoride in pediatric dentistry training programs: survey of graduate program directors. Pediatr Dent. 38(3):212-217.

16. Vasquez E, Zegarra G, Chirinos E, Castillo JL, Taves DR, Watson GE, Dills R, Mancl LL, Milgrom P. 2012. Short term serum pharmacokinetics of diammine silver fluoride after oral application. BMC Oral Health. 12:60.

17. Whitford GM. 1987. Fluoride in dental products: safety considerations. J Dent Res. 66(5):1056-1060. 